\title{
Plasma hydrodynamics in view of quasilinear effects
}

\author{
K. V. Gamayunov, ' G. V. Khazanov, ${ }^{2}$ E. N. Krivorutsky,' T. I. Gombosi ${ }^{2}$ and V. N. Oraevski ${ }^{3}$ \\ ' Altai State University, 656099 Barnaul, Russia \\ ${ }^{2}$ Space Physics Research Laboratory, University of Michigan, Ann Arbor, MI 48104, U.S.A. \\ ${ }^{3}$ IZMIRAN, 142092 Troitsk, Moscow Region, Russia
}

Received in final form 13 August 1992

\begin{abstract}
In this paper we have obtained expressions for the moments of the quasilinear integral of anisotropic plasma collisions. Resonant and adiabatic interactions of particles with an arbitrary wave mode are considered. Expressions for the moments describing the interactions of ion-cyclotron waves with ring current and magnetospheric helium ions have been derived as an example using obtained results.
\end{abstract}

\section{Introduction}

The hydrodynamical approach is widely used when describing the behavior of both cosmic and artificial plasmas. This approach makes it possible to analyze the behavior of multicomponent systems. In previous work, only interparticle collisions were considered (Oraevski et al., 1985).

Early treatments which only accounted for interparticle collisions had a fairly restricted sphere of applicability, because they did not consider wave-particle interactions which are particularly important in the ring current region of the Earth's magnetosphere. The ring current's interaction with the plasmasphere leads to intensive generation of ion-cyclotron waves (Cornwall, 1964 ; Kennel and Petschek, 1966). These waves were used to explain the effects of precipitation of ring current protons into the Earth's atmosphere (Cornwall et al., 1970), excitation of SARarcs (Cornwall et al., 1971), formation of the "hot zone" of the plasmasphere (Galeev, 1975) and many other phenomena.

The wave-particle interaction may be included if we add the corresponding moments of the quasilinear collision integral (Akhiezer, 1974) to the hydrodynamic equations. Similar attempts were made in recent articles (Konikov et al., 1989; Gamayunov et al., 1991; Gorbachev et al., 1992). The authors of these papers examined the interaction of the ion-cyclotron waves generated by hot anisotropic protons of the ring current with electrons and protons of the background plasma. These results cannot be employed to describe the interactions of other types of observed waves which interact with a multicomponent plasma.

In this paper, the system of hydrodynamic equations governing the behavior of a multicomponent anisotropic plasma will be supplemented by terms which take into account the quasilinear interactions of particles with arbitrary waves. Resonant quasilinear interaction of waves with particles (Konikov et al., 1989; Gamayunov et al., 1991) may be insufficient to describe a system. For example, both resonant and adiabatic interactions must be used to describe the interaction of Langmuir waves with electrons. Therefore, in the following, when obtaining the moments of the quasilinear collision integral, we will consider both resonant and adiabatic interactions.

\section{Quasilinear interaction in hydrodynamics : general relationships}

Let us consider the case of a weakly turbulent plasma. The expression for the quasilinear integral which considers both resonant and adiabatic interaction was obtained previously (Belikov et al., 1974). Since it will subsequently be used in a somewhat different form, we will outline its calculation. The following equations for the regular $\left(f^{\mathrm{R}}\right)$ and turbulent $\left(f^{(1)}\right)$ components of the distribution function are obtainable using a standard procedure (for more detail see Tsytovich, 1971):

$$
\begin{aligned}
\frac{\partial f_{\mathrm{a}}^{\mathrm{R}}}{\partial t}+\mathbf{v} \frac{\partial f_{\mathrm{a}}^{\mathrm{R}}}{\partial \mathbf{r}}+\frac{1}{m_{\mathrm{a}}}\left[\mathbf{F}_{\mathrm{a}}\right. & \left.+\frac{e_{\alpha}}{\mathrm{c}}\left(\mathbf{v} \mathbf{B}_{0}\right)\right] \frac{\partial f_{\mathrm{a}}^{\mathrm{R}}}{\partial \mathbf{v}} \\
& =-\frac{e_{\mathrm{a}}}{m_{\mathrm{a}}}\left[\mathbf{E}+\frac{1}{\mathrm{c}}(\mathbf{v B})\right] \frac{\partial f_{\mathrm{a}}^{(1)}}{\partial \mathbf{v}}, \\
\frac{\partial f_{\alpha}^{(1)}}{\partial t}+\mathbf{v} \frac{\partial f_{\alpha}^{(1)}}{\partial \mathbf{r}}+\frac{1}{m_{\alpha}}\left[\mathbf{F}_{\alpha}\right. & \left.+\frac{e_{\alpha}}{\mathrm{c}}\left(\mathbf{v} \mathbf{B}_{0}\right)\right] \frac{\partial f_{\alpha}^{(1)}}{\partial \mathbf{v}} \\
& =-\frac{e_{\alpha}}{m_{\alpha}}\left[\mathbf{E}+\frac{1}{\mathrm{c}}(\mathbf{v B})\right] \frac{\partial f_{\alpha}^{\mathrm{R}}}{\partial \mathbf{v}},
\end{aligned}
$$

where $F_{\alpha}$ is the regular component of force and, for 
brevity, the integrals of interparticle collisions are omitted on the right-hand side of equation (1). The regular force may include the gravity and electrical terms. We wrote the magnetic term in evident form. In the further description, the regular force is considered as constant on the timespace scale of the turbulent quantities.

Then, if we use an accompanying coordinate system moving with the mean velocity of particles of the type $\alpha$ in considering a point in time and space

$$
\left[\mathbf{u}(\mathbf{r}, t)=\frac{1}{N} \int \mathbf{v} f(\mathbf{r}, \mathbf{v}, t) \mathrm{d} \mathbf{v}, N=\int f(\mathbf{r}, \mathbf{v}, t) \mathrm{d} \mathbf{v}\right]
$$

then equation (2) takes the form (we will omit index $\alpha$ ):

$$
\begin{aligned}
\frac{\partial f^{(1)}\left(t^{\prime} \cdot \mathbf{r}^{\prime} \cdot \mathbf{v}^{\prime}\right)}{\partial t^{\prime}}+\mathbf{v}^{\prime} \frac{\partial f^{(1)}}{\partial \mathbf{r}^{\prime}} & +\frac{e}{m^{\prime} \mathrm{c}}\left[\mathbf{v}^{\prime} \mathbf{B}_{0}\right] \frac{\partial f^{(1)}}{\partial \mathbf{v}^{\prime}} \\
& =-\frac{e}{m^{\prime}}\left(\mathbf{E}^{\prime}+\frac{1}{\mathrm{c}}\left[\mathbf{v}^{\prime} \mathbf{B}^{\prime}\right]\right) \frac{\partial f^{\mathrm{R}}}{\partial \mathbf{v}^{\prime}} .
\end{aligned}
$$

The primes indicate that all quantities are taken in a coordinate system moving with the plasma. If one neglects the background plasma's inhomogeneity and nonstationarity. equation (3) has the following solution (axis $z \| \mathbf{B}_{0}$ ) (Akhiezer, 1974) :

$$
\begin{aligned}
f_{k^{\prime}}^{(\prime)} & =\exp \left(i \frac{k_{\perp}^{\prime} \mathbf{v}_{\perp}^{\prime}}{\omega_{B}^{\prime}} \sin \left(\varphi^{\prime}-\chi^{\prime}\right)\right) \\
& \times \sum_{n} \frac{\exp \left(-\mathrm{in} \varphi^{\prime}\right)}{2 \pi \mathrm{i}\left(\omega^{\prime}-\mathbf{k}_{\|}^{\prime} \mathbf{v}_{\|}^{\prime}-n \omega_{B}^{\prime}\right)} \\
& \times \int_{0}^{2 \pi} \mathrm{d} \varphi^{\prime \prime} \exp \left(-\mathrm{i} \frac{\mathbf{k}_{\perp}^{\prime} \mathbf{v}_{\perp}^{\prime}}{\omega_{B}^{\prime}} \sin \left(\varphi^{\prime \prime}-\chi^{\prime}\right)\right) \\
& \times \exp \left(\mathrm{in} \varphi^{\prime \prime}\right) \frac{e}{m^{\prime}}\left[\mathbf{E}_{\mathbf{k}}^{\prime}\left(1-\frac{\mathbf{k}^{\prime} \mathbf{v}^{\prime}}{\omega^{\prime}}\right)+\frac{\mathbf{k}^{\prime}\left(\mathbf{v}^{\prime} \mathbf{E}_{\mathbf{k}^{\prime}}^{\prime}\right)}{\omega^{\prime}}\right] \frac{\partial f^{\mathrm{R}}}{\partial \mathbf{v}^{\prime}} .
\end{aligned}
$$

where $\mathbf{k}$ is the wavevector, $\cos \chi^{\prime}=k_{\mathrm{v}}^{\prime} / k_{1}, \cos \varphi^{\prime}=r_{x}^{\prime} / r_{1}^{\prime}$, $\omega_{B}^{\dagger}=e B_{0}^{\dagger} m \mathrm{c}$.

Assuming that the mean velocities $\mathbf{u}(|\mathbf{u}| \ll c)$ on small scales are coordinate- and time-independent, the transformations (see e.g. Alexandrov et al.. 1988) :

$$
\begin{aligned}
& \omega^{\prime}=\omega-\mathbf{k u}, \quad \mathbf{k}^{\prime}=\mathbf{k}, \quad \mathbf{E}_{k_{t}}^{\prime}=\beta_{1,}(\mathbf{u}) \mathbf{E}_{k^{\prime}}, \\
& \beta_{\eta j}(\mathbf{u})=\frac{\omega-\mathbf{k} \mathbf{u}}{\omega} \delta_{3 l}+\frac{\mathbf{k}_{i} \mathbf{u}}{\omega} .
\end{aligned}
$$

where $\delta_{i j}$ is the Kronecker delta, permit equation (4) to be rewritten in a laboratory coordinate system:

$$
\begin{aligned}
f_{k}^{(1)}= & \hat{\mathbf{L}} \exp \left(\mathrm{i} \frac{k_{\perp} r_{1}^{\prime}}{\omega_{B}} \sin \left(\varphi^{\prime}-\chi\right)\right) \\
& \times \sum_{n} \frac{\exp \left(\mathrm{in} \varphi^{\prime}\right)}{2 \pi \mathrm{i}\left(\omega-\mathbf{k u}-k v^{\prime}-n \omega_{B}\right)} \\
& \times \int_{n}^{2 \pi} \mathrm{d} \varphi^{\prime \prime} \exp \left(-\mathrm{i} \frac{k_{\perp} v_{\perp}^{\prime}}{\omega_{B}} \sin \left(\varphi^{\prime \prime}-\chi\right)\right) \\
& \times \exp \left(\mathbf{i n} \varphi^{\prime \prime}\right){ }_{m}^{e}\left[\frac{\mathbf{E}_{k}(\mathbf{k u})-\mathbf{k}\left(\mathbf{u} \mathbf{E}_{k}\right)}{\omega}\right.
\end{aligned}
$$

$$
\left.+\mathbf{E}_{k}\left(1-\frac{\mathbf{k} \mathbf{v}^{\prime}}{\omega}\right)+\frac{\mathbf{k}\left(\mathbf{v}^{\prime} \mathbf{E}_{k}\right)}{\omega}\right] \frac{\partial f^{\mathbf{R}}}{\partial \mathbf{v}^{\prime}}
$$

Here $\hat{\mathbf{L}} g\left(\mathbf{v}^{\prime}\right) \equiv g(\mathbf{v}-\mathbf{u})$.

On substituting equation (6) into the right-hand side of equation (1), multiplying by $m h(\mathbf{v}-\mathbf{u})$ and integrating over velocity, we obtain the following expression for the moments of the quasilinear integral:

$$
\begin{aligned}
& M=\frac{e^{2}}{m} \int \mathrm{d} \mathbf{k} I_{\mathbf{k}}^{\sigma}(\mathbf{r}, t) \int \mathrm{d} \mathbf{v}\left\{\mathbf{d}_{\mathbf{k}}^{\sigma^{*}}-\frac{\mathbf{e}_{\mathbf{k}}^{\sigma^{*}}(\mathbf{k v})-\mathbf{k}\left(\mathbf{v} e_{\mathbf{k}}^{\sigma^{*}}\right)}{\omega_{\mathbf{k}}^{\sigma^{*}}}\right\} \frac{\partial h(\mathbf{v})}{\partial \mathbf{v}} \\
& \times \sum_{n} \frac{\exp (-\mathbf{i n} \varphi)}{2 \pi \mathrm{i}\left(\omega_{\mathbf{k}}^{\sigma}-\mathbf{k} \mathbf{u}-k_{\|} v_{\|}-n \omega_{B}\right)} \\
& \times \int_{0}^{2 \pi} \mathrm{d} \varphi^{\prime} \exp \left(-\mathbf{i} \frac{k_{\perp} v_{\perp}^{\prime}}{\omega_{B}} \sin \left(\varphi^{\prime}-\chi\right)\right) \\
& \times \exp \left(\mathbf{i n} \varphi^{\prime}\right)\left[\mathbf{d}_{\mathbf{k}}^{\sigma}-\frac{\mathbf{e}_{\mathbf{k}}^{\sigma}(\mathbf{k} \mathbf{v})-\mathbf{k}\left(\mathbf{v} \mathbf{e}_{\mathbf{k}}^{\sigma}\right)}{\omega_{\mathbf{k}}^{\sigma}}\right] \frac{\partial f^{\mathbf{R}}}{\partial \mathbf{v}},
\end{aligned}
$$

where primes were omitted for $\mathrm{v}^{\prime}$ and the asterisk refers to complex conjugation.

We make the following definitions:

$$
\mathbf{d}_{\mathbf{k}}^{\sigma}=\frac{\mathbf{e}_{\mathbf{k}}^{\sigma}\left(\omega_{\mathbf{k}}^{\sigma}-\mathbf{k u}\right)+\mathbf{k}\left(\mathbf{u} \mathbf{e}_{\mathbf{k}}^{\sigma}\right)}{\omega_{\mathbf{k}}^{\sigma}} ; \quad I_{\mathbf{k}}^{\sigma}(\mathbf{r}, t)=\left|E_{\mathbf{k}}^{\sigma}(\mathbf{r}, t)\right|^{2},
$$

where $\mathbf{e}_{\mathbf{k}}^{\sigma}$ is a polarization vector of the wave oscillations of type $\sigma$ and $f^{\mathrm{R}}$ refers to a distribution function in the reference frame moving with velocity $\mathbf{u}$.

By making the following definitions [cf. equation (7)],

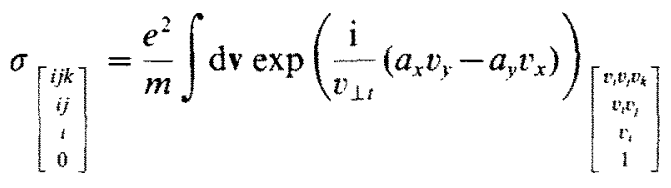

$$
\begin{aligned}
& \times \sum_{n} \frac{\exp (-\mathbf{i} n \varphi)}{2 \pi \mathrm{i}\left(\omega_{\mathbf{k}}^{\sigma}-\mathbf{k u}-k_{\|} \boldsymbol{v}_{\|}-n \omega_{B}\right)} \\
& \times \int_{0}^{2 \pi} \mathrm{d} \varphi^{\prime} \exp \left(-\mathrm{i} \frac{k_{\perp} v_{\perp}}{\omega_{B}} \sin \left(\varphi^{\prime}-\chi\right)+i n \varphi^{\prime}\right) \\
& \times\left[\mathbf{d}_{\mathbf{k}}^{\sigma}-\frac{\mathbf{e}_{\mathbf{k}}^{\sigma}(\mathbf{k v})-\mathbf{k}\left(\mathbf{v} \mathbf{e}_{\mathbf{k}}^{\sigma}\right)}{\omega_{\mathrm{k}}^{\sigma}}\right] \frac{\partial f^{\mathrm{R}}}{\partial \mathbf{v}} ; \\
& v_{\perp t}^{2}=\frac{T_{\perp}}{m} ; \quad(i, j, k)=x, y, z=1,2,3 \text {; }
\end{aligned}
$$

one may rewrite equation (7) in the form used in the hydrodynamical description (Oraevski et al., 1985), i.e. $h=v_{\alpha}, v_{\alpha} v_{\beta}, v_{\alpha} v_{\beta} v_{;}$, etc. :

$$
\begin{aligned}
& J_{\alpha}=\left.\int \mathrm{d} \mathbf{k} I_{\mathbf{k}}^{\sigma}\left(d_{\mathbf{k} \alpha}^{\sigma^{*}} \sigma_{0}-\frac{e_{\mathbf{k} \alpha}^{\sigma^{*}} k_{t}-k_{\alpha} e_{\mathbf{k} i}^{\sigma^{*}}}{\omega_{\mathbf{k}}^{\sigma^{*}}} \sigma_{t}\right)\right|_{a=k_{1} v_{\mu} / \omega_{B}}, \\
& J_{x ; \beta}=\int \mathrm{d} \mathbf{k} I_{\mathbf{k}}^{\sigma}\left[\left(d_{\mathbf{k} \alpha}^{\sigma^{*}} \sigma_{\beta}-\frac{e_{\mathbf{k} \alpha}^{\sigma^{*}} k_{i}-k_{\alpha} e_{\mathbf{k} i}^{\sigma^{*}}}{\omega_{\sigma_{k}^{*}}^{\sigma^{*}}} \sigma_{i \beta}\right)\right. \\
& +(\beta, \alpha)]\left.\right|_{\alpha=k_{1} v_{1 /} / \omega_{B}}
\end{aligned}
$$




$$
\begin{aligned}
& J_{\alpha \beta \gamma}=\int \mathrm{d} \mathbf{k} I_{\mathbf{k}}^{\sigma}\left[\left(d_{\mathbf{k} \alpha}^{\sigma_{\alpha}^{*}} \sigma_{\beta \gamma}-\frac{e_{\mathbf{k} \alpha}^{\sigma_{\alpha}^{*}} k_{i}-k_{\alpha} e_{\mathbf{k} i}^{\sigma^{*}}}{\omega_{\mathbf{k}}^{\sigma^{*}}} \sigma_{i \beta y}\right)\right. \\
& +[\gamma, \alpha, \beta]+[\beta, \gamma, \alpha]]_{a=k_{\perp} \nu_{\gamma}^{\prime} / \omega_{B}} .
\end{aligned}
$$

It is obvious that during the quasilinear interaction the number of particles is unchanged, i.e. for the $h=1$ moment, $J_{0}=0$. Here, the meaning of $\alpha, \beta, \gamma$ are any $x$, $y, z$. The notations $[\beta, \alpha],[\gamma, \alpha, \beta]$, etc. mean that the first term must be copied with substitution $\alpha \rightarrow \beta, \beta \rightarrow \alpha$ in equation (10); $\alpha \rightarrow \gamma, \beta \rightarrow \alpha, \gamma \rightarrow \beta$ in the second term and $\alpha \rightarrow \beta, \beta \rightarrow \gamma, y \rightarrow \alpha$ in the third term in equation (11).

For receiving equations (9)-(11), different functions $h$ $\left(h=v_{x}, v_{x} v_{\beta}, v_{x} v_{\beta} v_{1}\right.$, etc.) are replaced into equation $(7)$. Then. the derivative $\partial h / \partial v$, is multiplied by the bracket $\left\{\mathbf{d}_{\mathbf{k}}^{\sigma^{*}}-\left[\mathbf{e}_{\mathbf{k}}^{\sigma^{*}}(\mathbf{k v})-\mathbf{k}\left(\mathbf{v e}_{\mathbf{k}}^{\sigma^{*}}\right)\right] / \omega_{\mathbf{k}}^{\sigma^{*}}\right\}$, and the definitions (8) are used. We introduced parameter a which is equal to $k_{x} v_{\perp \ell} / \omega_{B}, k_{y} v_{\perp t} / \omega_{B}$ in the first exponential multiplier (8) in order to distinguish $k_{x}, k_{y}$ in this term from the others. It will be used later for determining the connection between $\sigma_{\alpha \beta}$ and $\sigma_{\alpha}, \sigma_{\alpha \beta \gamma}$ and $\sigma_{\alpha \beta *}$ etc. [expressions (13)(16)].

Equations (9)-(11) can be reduced to a simpler form if the relationships of continuity for the moments following from equation (3) are used. By introducing:

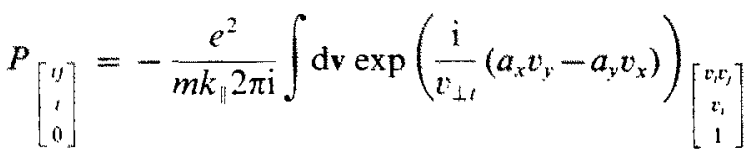

$$
\begin{aligned}
& \times \sum_{n} \exp (-i n \varphi) \int_{0}^{2 \pi} \mathrm{d} \varphi^{\prime} \exp \left(-i \frac{k_{\perp} v_{\perp}}{\omega_{B}} \sin \left(\varphi^{\prime}-\chi\right)\right) \\
& \times \exp \left(\operatorname{in} \varphi^{\prime}\right)\left\{\mathbf{d}_{\mathbf{k}}^{\sigma}-\frac{\mathbf{e}_{\mathbf{k}}^{\sigma}(\mathbf{k v})-\mathbf{k}\left(\mathbf{v} \mathbf{e}_{\mathrm{k}}^{\sigma}\right)}{\omega_{\mathrm{k}}^{\sigma}}\right\} \frac{\partial f^{\mathrm{R}}}{\partial \mathbf{v}},
\end{aligned}
$$

we get :

$$
\begin{aligned}
& J_{\alpha}=\int \mathrm{d} \mathbf{k} I_{\mathbf{k}}^{\sigma}\left\{\frac{k_{x}}{\omega_{\mathbf{k}}^{\sigma^{*}}}\left[\left(\mathbf{u} \mathbf{e}_{\mathbf{k}}^{\sigma^{*}}\right) \sigma_{0}+\mathbf{e}_{\mathbf{k} i}^{\sigma_{i}^{*}} \sigma_{i}\right]\right. \\
& \left.+e_{\mathbf{k} x}^{\sigma^{*}} \frac{\omega_{\mathbf{k}}^{\sigma^{* *}}-\omega_{\mathbf{k}}^{\sigma \sigma}}{\omega_{\mathbf{k}}^{\sigma^{*}}} \sigma_{0}\right\}\left.\right|_{a=k_{\perp} t_{1 / \gamma} / \omega_{B}}, \\
& J_{x \beta}=\int \mathrm{d} \mathbf{k} I_{\mathbf{k}}^{\sigma}\left\{\left[\frac{k_{\alpha}}{\omega \sigma_{\mathbf{k}}^{\sigma^{*}}}\left[\left(\mathbf{u} \mathbf{e}_{\mathbf{k}}^{\sigma^{*}}\right) \sigma_{\beta}+\mathbf{e}_{\mathrm{k},}^{\sigma^{*}} \sigma_{z \beta}\right]\right.\right. \\
& +e_{\mathbf{k}: x}^{\sigma^{*}} \frac{\omega_{\mathbf{k}}^{\sigma^{*}}-\omega_{\mathbf{k}}^{\pi}}{\omega_{\mathrm{k}}^{\sigma^{*}}} \sigma_{B}-\frac{k_{\mathrm{k}}}{\omega_{\mathrm{k}}^{\pi^{*}}} e_{\mathbf{k} x}^{\sigma^{*}} P_{B} \\
& \left.\left.-i \frac{\omega_{B}}{\omega_{\mathbf{k}}^{\sigma^{*}}} e_{\mathbf{k} x}^{\sigma^{*}}\left(\delta_{\beta 2} \sigma_{1}-\delta_{\beta 1} \sigma_{2}\right)\right]+[\beta, \alpha]\right\}\left.\right|_{a=k_{1} \boldsymbol{r}_{\mathrm{k}} ; \omega_{B}}, \\
& J_{x \beta_{i}}=\int \mathrm{d} \mathbf{k} I_{\mathbf{k}}^{\sigma}\left\{\left[\frac{k_{x}}{\omega_{\mathbf{h}}^{\sigma^{*}}}\left[\left(\mathbf{u} \mathbf{e}_{\mathbf{h}}^{\sigma^{*}}\right) \sigma_{\beta_{i}}+\mathbf{e}_{\mathbf{k} ;}^{\sigma^{*}} \sigma_{i \beta ;}\right]\right.\right. \\
& +e_{\mathrm{k} z}^{\sigma^{*}} \frac{\omega_{\mathrm{k}}^{\sigma^{*}}-\omega_{\mathrm{k}}^{\sigma}}{\omega_{\mathrm{k}}^{\sigma^{*}}} \sigma_{\beta_{j}}-\frac{k_{i}}{\omega_{\mathrm{k}}^{\sigma^{*}}} e_{\mathrm{k}: \alpha}^{\sigma^{*}} P_{\beta_{i}} \\
& \left.-\mathrm{i} \frac{\omega_{\beta}}{\omega_{k}^{\sigma^{*}}} e_{k z}^{\alpha^{*}}\left(\delta_{\beta 2} \sigma_{\eta \eta}-\delta_{\beta 1} \sigma_{2 y}+\delta_{i 2} \sigma_{1 \beta}-\delta_{z ;} \sigma_{2 \beta}\right)\right]
\end{aligned}
$$

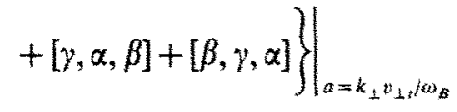

Using equations (8) and (12) we have the following:

$$
\begin{aligned}
& \sigma_{1}=\mathrm{i} v_{\perp t} \frac{\partial \sigma_{0}}{\partial a_{y}} ; \quad \sigma_{2}=-\mathrm{i} v_{\perp t} \frac{\partial \sigma_{0}}{\partial a_{x}} \\
& \sigma_{3}=P_{0}+\frac{\omega_{\mathrm{k}}^{\sigma^{\prime}}}{k_{\|}} \sigma_{0}+\mathrm{i} \frac{\omega_{B}}{k_{3}}\left[a_{y} \frac{\partial}{\partial a_{x}}-a_{x} \frac{\partial}{\partial a_{y}}\right] \sigma_{0} \\
& \sigma_{1 x}=i v_{1 t} \frac{\partial \sigma_{x}}{\partial a_{y}} ; \quad \sigma_{2 x t}=-i v_{1 /} \frac{\partial \sigma_{z}}{\partial a_{x}} ; \\
& \sigma_{3\}}=P_{3}+\frac{\omega_{\mathbf{k}}^{\sigma^{*}}}{k_{1}} \sigma_{3}+\mathrm{i} \frac{\omega_{B}}{k_{3}}\left[a_{y} \frac{\partial}{\partial a_{x}}-a_{x} \frac{\partial}{\partial a_{y}}\right] \sigma_{3} ; \\
& \sigma_{1 \beta \gamma}=\mathrm{i} v_{\perp} \frac{\partial \sigma_{\beta \gamma}}{\partial a_{y}} ; \quad \sigma_{2 \beta \gamma}=-\mathrm{i} v_{\perp, t} \frac{\partial \sigma_{\beta \gamma}}{\partial a_{x}} ; \\
& \sigma_{333}=P_{33}+\frac{\omega_{\mathbf{k}}^{f^{\prime}}}{k_{\sharp}} \sigma_{33}+\mathrm{i} \frac{\omega_{B}}{k_{1}}\left[a_{v} \frac{\partial}{\partial a_{x}}-a_{x} \frac{\partial}{\partial a_{y}}\right] \sigma_{33} .
\end{aligned}
$$

The relations (13)-(15) may be verified by the direct substitution of expressions (8) and (12) in these formulae.

If we use the following relationships:

$$
\begin{aligned}
& P_{1}=\mathrm{i} v_{\perp t} \frac{\partial P_{0}}{\partial a_{y}} ; \quad P_{2}=-\mathrm{i} v_{\perp t} \frac{\partial P_{0}}{\partial a_{x}} ; \\
& P_{1 \alpha}=\mathrm{i} v_{\perp t} \frac{\partial P_{\alpha}}{\partial a_{v}} ; \quad P_{2 \alpha}=-\mathrm{i} v_{\perp t} \frac{\partial P_{\alpha}}{\partial a_{x}},
\end{aligned}
$$

then, when calculating the functions $P$, one can put $\mathbf{a}=\mathbf{k}_{\perp} v_{\perp d} / \omega_{B}$ :

$$
\begin{aligned}
& \left.P_{\theta}\right|_{\mathbf{z}=\mathbf{k}_{1} \mathrm{r}_{1 ;} l \omega_{B}}=0 \text {, }
\end{aligned}
$$

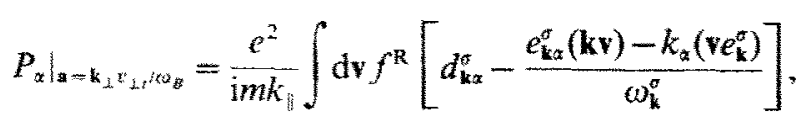

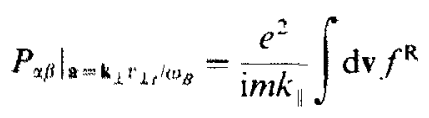

$$
\begin{aligned}
& \times\left\{v_{\alpha}\left[d_{\mathbf{k} \beta}^{\sigma}-\frac{e_{\mathbf{k} \beta}^{\sigma}(\mathbf{k v})-k_{\beta}\left(\mathbf{v} e_{\mathbf{k}}^{\alpha}\right)}{\omega_{\mathbf{k}}^{\sigma}}\right]+[\beta, \alpha]\right\} .
\end{aligned}
$$

Thus, we have written the expressions for the moments of the quasilinear integral of collisions in terms of the function $\sigma_{0}$ (its physical meaning is clear from its relationship to the charge density of $\alpha$ particles: $\rho_{\alpha}^{\prime}=$ $\left.\left.\sigma_{0}^{x} E_{\mathrm{k}}^{\prime}\right|_{\mathrm{z}=k_{1} r_{1} ; \omega_{n}}\right)$ and $P$. The hydrodynamic equations should be supplemented by an expression governing the energy density evolution of the plasma oscillations. The resulting system takes into account both the resonant and adiabatic quasilinear interactions.

\section{Moments of the quasilinear integral of collisions for a bi-Maxwellian plasma}

By virtue of the assumption made above [see equation (4)], the distribution function $f^{R}$ can depend on coor- 
dinates and on time [in expressions (8) and (12)] only as the parameters. If, moreover, interparticle collisions dominate and there is no need to consider plateau formation on $f^{R}$, the following expression (in the plasma frame) is often used:

$$
f^{\mathrm{R}}(\mathrm{v})=\frac{N_{\alpha}}{(2 \pi)^{3 / 2} v_{\perp,}^{2} v_{\| t}} \exp \left(-\frac{v_{\|}^{2}}{2 v_{\| t}^{2}}-\frac{v_{\perp}^{2}}{2 v_{1 t}^{2}}\right),
$$

where $N_{\alpha}$ is the density of $\alpha$ particles and $v_{\|}, v_{1,}$ are longitudinal (along the external magnetic field) and transverse thermal velocities, respectively. The density and temperatures in equation (20) depend parametrically on $r$ and $t$. Note that the calculation of moments of the quasilinear integral of collisions using equation (20) does not contradict the fact that, on scales in excess of the scale of averaging in equation (1), the distribution function can contain dissipative terms (for example, the Grad approximation).

In the case of Coulomb collisions, the question of the consistency of the approximations, in terms of which the cross-sections and moments of the integral of collisions are calculated, does not arise.

On substituting equation (20) into equation (8) and integrating over the velocity, we obtain:

$$
\begin{aligned}
\sigma_{0}= & \frac{e^{2} N}{\mathrm{i} m v_{\perp t} \omega_{\mathbf{k}}^{\sigma}} \sum_{n} \exp \left(\mathrm{in}\left(\chi-\chi^{\prime}\right)\right)\left\{\exp \left(-\frac{\alpha^{2}+\lambda^{2}}{2}\right) I_{n}(a \lambda)\right. \\
& \times\left[\frac{\omega_{\mathbf{k}}^{\sigma}}{k_{\|} v_{\perp t}} d_{\mathbf{k} \|}^{\sigma}(A+1)\left(1+\mathrm{i} \sqrt{\pi} z_{n} w\left(z_{n}\right)\right)\right. \\
& +\frac{n}{\lambda}\left[\frac{\mathrm{d}_{\mathbf{k} \perp}^{\sigma} \mathbf{k}_{\perp}}{k_{\perp}} \mathrm{i} \sqrt{\pi} z_{0} w\left(z_{n}\right)+A\left(\frac{\mathbf{e}_{\mathbf{k} \perp}^{o} \mathbf{k}_{\perp}}{k_{\perp}}-e_{\mathbf{k} \|}^{\sigma} \frac{k_{\perp}}{k_{\|}}\right)\right. \\
& \left.\left.\times\left(1+\mathrm{i} \sqrt{\pi} z_{n} w\left(z_{n}\right)\right)\right]\right] \\
& +\mathrm{i} \lambda \exp \left(-\frac{\alpha^{2}+\lambda^{2}}{2}\right)\left[-I_{n}(a \lambda)+\frac{a}{\lambda} I_{n}^{\prime}(a \lambda)\right] \\
& \left.\times\left[\frac{\left(\mathbf{k d}_{\mathbf{k}}^{\sigma}\right)_{\|}}{k_{\perp}} \mathrm{i} \sqrt{\pi} z_{0} w\left(z_{n}\right)+\frac{\left(\mathbf{k} \mathbf{e}_{\mathbf{k}}^{\sigma}\right)_{i}}{k_{\perp}} A\left(1+\mathrm{i} \sqrt{\pi} z_{n} w\left(z_{n}\right)\right)\right]\right\} .
\end{aligned}
$$

The designations used here are

$$
\begin{aligned}
\tan \chi^{\prime} & =\frac{a_{y}}{a_{x}} ; \quad \lambda=\frac{k_{\perp} v_{\perp t}}{\omega_{B}} ; \quad A=\frac{T_{\perp}}{T_{\|}}-1 ; \\
z_{n} & =\frac{\omega_{\mathbf{k}}^{\sigma^{\prime}}-n \omega_{B}}{\sqrt{2} k_{\|} v_{\| t}} ; \quad z_{0}=\frac{\omega_{\mathbf{k}}^{\sigma}}{\sqrt{2} k_{\|} v_{\| t}} ;
\end{aligned}
$$

$I_{n}$ is the modified Bessel's function and $w\left(z_{n}\right)$ is the probability integral of a complex argument:

$$
w\left(z_{n}\right)=\exp \left(-z_{n}^{2}\right)\left[1+\frac{2 \mathrm{i}}{\sqrt{\pi}} \int_{0}^{z_{m}} \exp \left(t^{2}\right) \mathrm{d} t\right] .
$$

The expressions for the functions $P$ [equation (12)] assume the form:

$$
\begin{gathered}
\left.P_{\alpha}\right|_{a=k_{1} v_{\perp i} / \omega_{s}}=\frac{\omega_{\mathrm{p}}^{2}}{4 \pi i k_{\|}} d_{\mathrm{k} \alpha}^{\sigma} ; \\
\left.P_{\alpha \beta}\right|_{a=k_{\perp} v_{\perp \lambda} / \omega_{B}}=\frac{\omega_{\mathrm{p}}^{2}\left(e_{\mathrm{k} \alpha}^{\sigma} k_{\beta}-e_{\mathrm{k} \beta}^{\sigma} k_{\alpha}\right)}{4 \pi \mathrm{i} k_{\|} \omega_{\mathrm{k}}^{\sigma}} \int \mathrm{dv}\left(v_{\alpha}^{2}-v_{\beta}^{2}\right) f^{\mathrm{R}},
\end{gathered}
$$

where $\omega_{\mathrm{p}}^{2}=4 \pi N_{\alpha} e^{2} / m$ is the plasma frequency of the considered particles. It is most convenient to calculate the derivatives of $\sigma_{0}$ in parameters $a_{x}$ and $a_{y}$ in a cylindrical coordinate system. In this case, as follows from equation (21), $\left(\partial / \partial x^{\prime}\right)^{l}=(-\mathrm{in})^{l}$, and the relationships:

$$
\left.\lambda \frac{\partial}{\partial a} Q_{n}\right|_{a=k_{1} v_{1 ;} / \omega_{s}}=\lambda^{2}\left[\exp \left(-\lambda^{2}\right) I_{n}\left(\lambda^{2}\right)\right]^{\prime} \equiv x A_{n}^{\prime}(x),
$$

$$
\begin{gathered}
\left.\lambda^{2} \frac{\partial^{2}}{\partial a^{2}} Q_{n}\right|_{a=k_{1} v_{\perp} / \omega_{B}}=x^{2} A_{n}^{\prime \prime}(x)-x A_{n}(x), \\
\left.\lambda^{3} \frac{\partial^{3}}{\partial a^{3}} Q_{n}\right|_{a=k_{\perp} v_{1 /} / \omega_{B}}=x^{3} A_{n}^{\prime \prime \prime}(x)-3 x^{2} A_{n}^{\prime}(x), \\
\left.\lambda \frac{\partial}{\partial a} R_{n}\right|_{a=k_{\perp} v_{1 /} / \omega_{B}}=x A_{n}^{\prime \prime}(x)+\exp (-x) I_{n}^{\prime}(x),
\end{gathered}
$$

$\left.\lambda^{2} \frac{\partial^{2}}{\partial a^{2}} R_{n}\right|_{a=k_{\perp} v_{\perp i} / \omega_{s}}$

$$
=x^{2} A_{n}^{\prime \prime \prime}(x)+x A_{n}^{\prime \prime}(x)+x\left[\exp (-x) I_{n}^{\prime}(x)\right]^{\prime}
$$

and :

$$
\begin{aligned}
& \left.\lambda^{3} \frac{\partial^{3}}{\partial a^{3}} R_{n}\right|_{a=k_{\perp}{ }_{\perp} / \omega_{B}} \\
& \quad=x^{3} A_{n}^{\prime V}(x)+3 x^{2} A_{n}^{\prime \prime \prime}(x)-3 x \exp (-x) I_{n}^{\prime}(x),
\end{aligned}
$$

are valid, where :

$$
Q_{n}=\exp \left(-\frac{a^{2}+\lambda^{2}}{2}\right) I_{n}(a \lambda)
$$

and

$$
R_{n}=\exp \left(-\frac{a^{2}+\lambda^{2}}{2}\right)\left[-I_{n}(a \lambda)+\frac{a}{\lambda} I_{n}^{\prime}(a \lambda)\right]
$$

Using expressions (21)-(28), one can rewrite the moments of the quasilinear integral [equations (9)-(11)] for the case under consideration. The resulting expressions are rather unwieldy; however, for a number of particular situations (small Larmor radius, absence of transverse drift, resonant interaction, etc.), they simplify substantially.

\section{Moments of the quasilinear integral of collisions in the absence of a transverse drift}

If $\mathbf{u}=0$, then neither $I_{\mathbf{k}}^{\sigma}$, nor $\sigma_{0}$, nor the derivatives on $x^{\prime}$ and $a$ (after identifying $\mathbf{a}=\mathbf{k}_{\perp} v_{1 t} / \omega_{B}$ ), depend on $x$, and this allows us to simplify expressions (9)-(11). We choose the polarization vector in the form (Akhiezer, 1974):

$$
\mathbf{e}_{\mathrm{k}}^{\sigma}=\frac{1}{\left|g^{\sigma}\right|}\left\{\cos \chi+\mathrm{i} T^{\sigma} \sin \chi, \sin \chi-\mathrm{i} T^{\sigma} \cos \chi, e_{3}^{\sigma}\left|g^{\sigma}\right|\right\},
$$


where $T^{\sigma}$ is a combination of the components of the tensor of dielectric permeability $\varepsilon_{i j}\left(\omega_{\mathbf{k}}^{\sigma}, \mathbf{k}\right) ; \varepsilon_{3} \equiv \varepsilon_{z z}\left(\omega_{\mathbf{k}}^{\sigma}, \mathbf{k}\right)$ and $\left|q^{\sigma}\right|$ is the normalization. Now we can integrate expressions $\left(9^{\prime}\right)-\left(11^{\prime}\right)$ over $\chi$. Then :

$$
\begin{aligned}
& J_{\alpha}=\delta_{\alpha 3} \int \mathrm{d} \mathbf{k} I_{\mathbf{k}} \frac{1}{\omega_{k}^{*}} \sum_{n}\left[e_{\mathbf{k} \|}^{*}\left(\omega_{k}^{*}-n \omega_{B}\right)\right. \\
& \left.+\frac{k_{\|} \omega_{B}}{k_{\perp}|g|}\left(T^{*} \lambda \frac{\partial}{\partial a}+n\right)\right]\left.\sigma_{0}^{n}\right|_{a=k_{\perp} n_{\perp l} ; \omega_{B}} \\
& J_{\alpha \beta}=\int \mathrm{d} \mathbf{k} I_{\mathbf{k}} \frac{1}{\omega_{\mathbf{k}}^{*} k_{\|}}\left\{\left(\delta_{\alpha 1} \delta_{\beta 1}+\delta_{\alpha 2} \delta_{\beta 2}-2 \delta_{\alpha 3} \delta_{\beta 3}\right) \frac{\omega_{\mathrm{p}}^{2}}{4 \pi \mathrm{i}}\right. \\
& \times\left[\left(\mathrm{ke}_{\mathrm{k}}^{*}\right) \mathbf{d}_{\mathrm{k}}\right]_{1} \\
& +\left(\delta_{x \mid} \delta_{\beta 1}+\delta_{x 2} \delta_{\beta 2}\right) \sum_{n}\left[n \omega_{B} e_{\mathbf{k} \|}^{*}\left(\omega_{\mathbf{k}}-n \omega_{B}\right)\right. \\
& \left.+\left(n \omega_{B}+\omega_{\mathrm{k}}^{*}-\omega_{\mathrm{k}}\right) \frac{\omega_{B} k_{\|}}{k_{\perp}|g|}\left(T^{*} \lambda \frac{\partial}{\partial a}+n\right)\right] \sigma_{0}^{n}+2 \delta_{\alpha 3} \delta_{\beta 3} \\
& \times \sum_{n}\left(\omega_{\mathbf{k}}^{\prime}-n \omega_{B}\right)\left[e_{\mathbf{k} \|}^{*}\left(\omega_{\mathbf{k}}^{*}-n \omega_{B}\right)\right. \\
& \left.\left.+\frac{\omega_{B} k_{1}}{k_{\perp}|g|}\left(T^{*} \dot{\lambda} \frac{\partial}{\partial a}+n\right)\right] \sigma_{0}^{n}\right\}\left.\right|_{a-k_{1} \varepsilon_{1} l^{* \omega_{B}}} \\
& J_{\alpha \beta \gamma}=\int \mathrm{d} \mathbf{k} I_{\mathbf{k}} \frac{1}{\omega_{\mathbf{k}}^{*} k_{\mathbf{k}}^{2}}\left\langle-\left[\delta_{\alpha 3}\left(\delta_{\beta 1} \delta_{\gamma 1}+\delta_{\beta 2} \delta_{\gamma 2}\right)\right.\right. \\
& +\delta_{\beta 3}\left(\delta_{; 1} \delta_{x 1}+\delta_{; 2} \delta_{x 2}\right) \\
& \left.+\delta_{; 3}\left(\delta_{x 1} \delta_{\beta 1}+\delta_{x 2} \delta_{\beta 2}\right)-3 \delta_{\alpha 3} \delta_{\beta 3} \delta_{\gamma 3}\right] \\
& \times \frac{\omega_{\mathrm{p}}^{2}}{4 \pi \mathrm{i}}\left[\frac{k_{\|} v_{\| t}^{2}}{\omega_{\mathbf{k}}} A\left|\left(\mathbf{e}_{\mathbf{k}}^{*} \mathbf{k}\right)_{\perp}\right|^{2}+\mathrm{i} \omega_{B}\left(\mathbf{e}_{\mathbf{k}}^{*} \mathbf{k}\right)_{\perp} \mathbf{d}_{\mathbf{k} \perp}\right] \\
& -3 \delta_{x 3} \delta_{\beta 3} \delta_{; 3} \frac{\omega_{p}^{2}}{4 \pi \mathrm{i}}\left\{\omega_{\mathrm{k}}^{\prime}\left[\left(\mathbf{k} \mathbf{e}_{\mathrm{k}}^{*}\right) \mathbf{d}_{\mathbf{k}}\right]_{\|}\right. \\
& \left.-\omega_{\mathbf{k}}^{*} k_{\|}\left|e_{\mathbf{k} \mid}\right|^{2}+\omega_{\mathbf{k}}^{*} e_{\mathbf{k} !}^{*} \mathbf{k}_{\perp} \mathbf{d}_{\mathbf{k} \perp}\right\}+3 \delta_{z 3} \delta_{\beta 3} \delta_{i 3} \sum_{n}\left(\omega_{\mathbf{k}}^{\prime}-n \omega_{B}\right)^{2} \\
& \times\left[\left(\omega_{\mathbf{k}}^{*}-n \omega_{B}\right) e_{\mathbf{k}}^{*}+\frac{\omega_{B} k_{1}}{k_{\perp}|g|}\left(T^{*} \lambda \frac{\hat{c}}{\partial a}+n\right)\right] \sigma_{0}^{n} \\
& +\left[\delta_{\alpha 3}\left(\delta_{\beta 1} \delta_{; 1}+\delta_{\beta 2} \delta_{i 2}\right)+\delta_{\beta 3}\left(\delta_{; 1} \delta_{x 1}+\delta_{; 2} \delta_{x 2}\right)\right. \\
& \left.+\delta_{;}\left(\delta_{x 1} \delta_{\beta 1}+\delta_{\alpha 2} \delta_{\beta 2}\right)\right] \sum_{n}\left(\omega_{k}^{\prime}-n \omega_{B}\right)\left[n \omega_{B} e_{k}^{*}\left(\omega_{k}-n \omega_{B}\right)\right. \\
& \left.+\left(n \omega_{B}+\omega_{\mathbf{k}}^{*}-\omega_{\mathbf{k}}\right) \frac{\omega_{B} k_{1}}{k_{\perp}|g|}\left(T^{*} \dot{\partial} \frac{\hat{c}}{\partial a}+n\right)\right] \sigma_{0}^{n} \\
& -\left[\delta_{x 3}\left(\delta_{\beta 1} \delta_{y 1}+\delta_{\beta 2} \delta_{\gamma^{2}}\right)+\delta_{\beta 3}\left(\delta_{i 1} \delta_{x 1}+\delta_{\gamma 2} \delta_{\alpha 22}\right)\right. \\
& \left.+\delta_{\gamma 3}\left(\delta_{\alpha 1} \delta_{\beta 1}+\delta_{\alpha 2} \delta_{\beta 2}\right)\right] \\
& \times \frac{\omega_{B}^{2} k_{\|}^{2}}{2 k_{\perp}^{2}} \sum_{n}\left\{e_{\mathbf{k} \|}^{*}\left(\omega_{\mathbf{k}}^{*}-n \omega_{B}\right)\left(\lambda^{2} \frac{\partial^{2}}{\partial \alpha^{2}}+\lambda \frac{\partial}{\partial \alpha}-n^{2}\right)\right.
\end{aligned}
$$

$$
\begin{aligned}
& +\frac{\omega_{B} k_{\|}}{k_{\perp}|g|}\left[T^{*}\left(\lambda^{3} \frac{\partial^{3}}{\partial \alpha^{3}}+\hat{\lambda}^{2} \frac{\partial^{2}}{\partial \alpha^{2}}-\left(1+n^{2}\right) \lambda \frac{\partial}{\partial \alpha}+2 n^{2}\right)\right. \\
& \left.\left.+n\left(\lambda^{2} \frac{\partial^{2}}{\partial \alpha^{2}}+\lambda \frac{\partial}{\partial \alpha}-n^{2}\right)\right]\right\} \sigma \\
& +\frac{\omega_{p}^{2}}{4 \pi \mathrm{i}}\left[\delta_{\alpha 3}\left(\delta_{\beta 1} \delta_{\gamma 1}+\delta_{\beta 2} \delta_{\gamma 2}\right)+\delta_{\beta 3}\left(\delta_{\gamma 1} \delta_{\alpha 1}+\delta_{y 2} \delta_{\alpha 2}\right)\right. \\
& \left.+\delta_{73}\left(\delta_{\alpha 1} \delta_{\beta 1}+\delta_{\alpha 2} \delta_{\beta 2}\right)\right] \\
& \left.\times\left\{k_{\mid 1} \omega_{\mathbf{k}}^{*} \mathbf{e}_{\mathbf{k} \perp}^{*} \mathbf{d}_{\mathbf{k} \perp}+\omega_{\mathbf{k}}\left[\left(\mathbf{k e}_{\mathbf{k}}^{*}\right) \mathbf{d}_{\mathbf{k}}\right]_{\|}\right\}\right)\left.\right|_{a=k_{\perp} v_{\perp} / \omega_{\beta}}
\end{aligned}
$$

where $\sigma_{0}$ has the form:

$$
\begin{aligned}
\sigma_{0}= & \sum_{n} \sigma_{0}^{n}=\frac{\omega_{p}^{2}}{4 \pi \mathrm{i} v_{\perp t} \omega_{\mathbf{k}}} \\
& \times \sum_{n}\left\langle\exp \left(-\frac{a^{2}+\lambda^{2}}{2}\right) I_{n}(a \lambda) e_{\mathbf{k} \mid}\right. \\
& \times\left\{\frac{\omega_{\mathbf{k}}}{k_{\mid} v_{\perp t}}\left[1+A\left(1-\frac{n \omega_{B}}{\omega_{\mathbf{k}}^{o}}\right)\right]\left[1+\mathrm{i} \sqrt{\pi} z_{n} w\left(z_{n}\right)\right]\right. \\
& \left.+\frac{n \omega_{B}}{\omega_{\mathbf{k}}} \frac{u_{\|}}{i_{\perp t}} \mathrm{i} \sqrt{\pi z} w\left(z_{n}\right)\right\}+\frac{\lambda}{|g|}\left\{\mathrm{i} \sqrt{\pi} z_{0} w\left(z_{n}\right)\right. \\
& +A\left[1+\mathrm{i} \sqrt{\pi} z_{n} w\left(z_{n}\right)\right\} \exp \left(-\frac{a^{2}+\lambda^{2}}{2}\right) \\
& \left.\times\left\{I_{n}(a \lambda) \frac{n}{\lambda^{2}}+T\left[-I_{n}(a \lambda)+\frac{a}{\lambda} I_{n}^{\prime}(a \lambda)\right]\right\}\right\rangle .
\end{aligned}
$$

When substituting the derivatives $(\partial / \partial a)^{l}$ into equations (30)-(32), it is more convenient to use, not equations (23)-(28), but their combinations. Expressions for these combinations are given in the Appendix.

Thus, we have obtained the moments of the quasilinear collision integral which take into account the resonant and adiabatic interactions of arbitrary types of oscillations with arbitrary plasma particles. Expressions $\left(9^{\prime}\right)-\left(11^{\prime}\right)$, (13)-(17). (21) and (22) are suitable for describing the plasma in the field of arbitrarily directed regular force $\mathbf{F}_{\alpha}$, or equations (30)-(33) in the absence of a transverse drift.

As mentioned above, the system of hydrodynamic equations, taking into account the contributions from the quasilinear interaction, should be solved together with the equation describing the evolution of the spectral energy of waves $I_{h}^{\sigma}$. However, as a first approximation, we can use the energy spectra of the given type of waves registered on satellites in near-terrestrial space.

In conclusion we will derive the expressions for the moment of the quasilinear integral of collisions which can be used when modelling the interactions of ion-cyclotron waves with ions in the ring current region.

As we mentioned in the Introduction, the anisotropic protons of the ring current generate intensive left-hand polarized ion-cyclotron waves in the region of the plasmasphere. Moreover, the generation takes place during the cyclotron interaction. Using equations (30)-(33) and equations (A1)-(A6) for $n=1$, assuming $\lambda \ll 1$ and neglecting $e_{k \mid}$, we get fairly simple expressions which take 
into account the interaction of ring current protons and ion-cyclotron waves:

$$
\begin{aligned}
& J_{\alpha}=\delta_{\alpha 3} \frac{\omega_{p}^{2}}{8 \sqrt{\pi}} \int \mathrm{d} \mathbf{k} I_{\mathrm{k}} \frac{k_{\|}}{\omega_{\mathrm{R}}^{2}|g|^{2}}|1+T|^{2} \\
& \times\left(z_{0}+A z_{1}\right) \exp \left(-z_{1}^{2}\right) \operatorname{sign} k_{n}, \\
& J_{\alpha \beta}=\frac{\omega_{p}^{2} \omega_{B}}{8 \sqrt{\pi}} \int \mathrm{d} \mathbf{k} I_{\mathbf{k}} \frac{|1+T|^{2}}{\omega_{\mathrm{R}}^{2}|g|^{2}} \\
& \times\left[\delta_{\alpha 1} \delta_{\beta 1}+\tilde{\delta}_{\alpha 2} \delta_{\beta 2}+2 \delta_{\alpha 3} \tilde{\delta}_{\beta 3}\left(\frac{\omega_{\mathrm{R}}^{\prime}}{\omega_{B}}-1\right)\right] \\
& \times\left(z_{0}+A z_{1}\right) \exp \left(-z_{1}^{2}\right) \operatorname{sign} k_{1} \text {, } \\
& J_{\alpha p_{\gamma}}=\frac{\omega_{p}^{2} \omega_{R}^{2}}{8 \sqrt{\pi}} \int \mathrm{d} \mathbf{k} I_{\mathbf{k}} \frac{\| 1+\left.T\right|^{2}}{\omega_{R}^{2} k_{\|}|g|^{2}} \\
& \times\left\{\left[\delta_{x 3}\left(\delta_{\beta 1} \delta_{\gamma^{1} 1}+\delta_{\beta 2} \delta_{y^{2}}\right)+\delta_{\beta 3}\left(\delta_{y, 1} \delta_{\alpha 1}+\delta_{y 2} \delta_{x 2}\right)\right.\right. \\
& \left.+\delta_{\gamma 3}\left(\delta_{x \mid} \delta_{\beta 11}+\delta_{* 2} \delta_{\beta 2}\right)\right]\left[\frac{\omega_{\mathrm{R}}^{r}}{\omega_{\beta}}-1+\frac{2 k_{\beta}^{2} v_{1,}^{2}}{\omega_{B}^{2}}\right] \\
& \left.+3 \delta_{\alpha 3} \delta_{\beta 3} \delta_{\gamma 3}\left(\frac{\omega_{\mathrm{R}}^{i}}{\omega_{\beta}}-1\right)^{2}\right\} \\
& \times\left(z_{0}+A z_{1}\right) \exp \left(-z_{1}^{2}\right) \operatorname{sign} k_{\text {. }}
\end{aligned}
$$

where:

$$
\begin{gathered}
\omega_{\mathrm{R}}=\operatorname{Re}\left(\omega_{\mathrm{k}}\right) ; \quad \omega_{\mathrm{R}}^{\prime}=\omega_{\mathrm{R}}-k_{1} u_{1} \\
z_{1}=\frac{\omega_{\mathrm{R}}^{\prime}-\omega_{B}}{\sqrt{2} k_{k} v_{k_{t}}} ; \quad T=\frac{\mathrm{i} \varepsilon_{\mathrm{rt}}}{\varepsilon_{\lambda_{2}}-N^{2}} ; \quad N^{2}=\frac{k^{2} \mathrm{c}^{2}}{\omega_{k}^{2}} .
\end{gathered}
$$

The tensor of dielectric permeability of the cold magnetized plasma in previous expressions may be used.

If there is a small addition of helium ions to the ring current region, then the dispersive dependence of ioncyclotron waves is greatly modified (Young et al., 1981). The presence of helium leads to three branches in the dispersion relation for oblique propagation of waves. The first branch (class I) is left-hand polarized and lies below the gyrofrequency of helium ions $\mathrm{Hc}^{+}$(Korth et al. 1984). The interaction of such waves with $\mathrm{He}^{-m}$ will also be described by expressions (34) (36) with substitution of the anisotropic proton parameters for the parameters of $\mathrm{He}^{+}$.

In conclusion. we should like to stress that the moments of the quasilinear collision integrals obtained in this paper take into account both resonant and adiabatic interactions of arbitrary waves and particles. It gives us an opportunity to use the system of the hydrodynamical equations for investigation of multicomponent plasma in which the developed wave activity takes place.

The obtained results include. as a particular case. the outcome of previous papers (Konikov et al., 1989: Gamayunovet al., 1991 : Gorbachev et al. 1992) and may be useful for a hydrodynamical description of the solar wind, plasmas of the other planets and so on.

Acknowledgement-This work was supported by the NASA Grant Nos. NAGW-1619 and NAG5-1500, as well as NSF Grant ATM-9114409. We thank the referee for his assistance in evaluating this paper.

\section{References}

Akhiezer, A. 1., (Editor) Plasma Electrodynamies. Nauka, Moscow, 1974

Alexandrov, A. F.. Bogdankevich, L. S. and Rukhadze, A. A.. Fundamentals of Electrodynamics of Plasma. Nauka, Moscow, 1988.

Belikov, V. S., Kolesnichenko, Ya. I. and Oraevski, V. N., Nonlinear theory of the thermonuclear Alfven plasma instability. Sow. Phys. JETP 39, 828, 1974.

Cornwall, J. M., Cyclotron instabilities and electromagnetic emission generation mechanisms. $J$. geophys. Res. 69, 4515 , 1964.

Cornwall, J. M., Coronity, F. V. and Thorne, R. M., Turbulent loss of ring current protons. J geophys. Res. 75, 4699, 1970.

Cornwall, J. M. Coronity, $F_{*} V_{*}$ and Thorne, R. M., Unified theory of SAR-arc formation at plasmapause. $J$. geophys. Res. 76, 4428, 1971 .

Galeev, A. A., Plasma turbulence in magnetosphere with special regard to plasma heating. In Physics of the Hot Plasma in the Magnetosphere. Plenum Press, London, 1975.

Gamayunov, K. V.. Krivorutsky, E. N. and Khazanov, G. V., Hydrodynamic description of magnetosphere plasma with due regard to the wave activity of Alfven and fast magnetosonic waves. Planet. Space Sci. 39, 1097, 1991 .

Gorbachev, O. A., Khazanov, G. V., Gamayunov, K. V. and Krivorutsky, E. N., A theoretical model for the ring current interaction with the Earth"s plasmasphere. Planet. Space Sci. 40, 859, 1992.

Kennel, C. F. and Petschek, H. E* Limit on stably trapped particle fluxes. J. geophys. Res. 71, 1, 1966.

Konikov, Yu. V., Gorbachev, O. A., Khazanov, G. V. and Chernov, A. A., Hydrodynamical equation for thermal electrons taking into account their scattering on ion-cyclotron waves in the outer plasmasphere of the Earth. Planet. Space Sci. 37, 1157, 1989 .

Korth, A., Kremser, $G_{*}$, Perraut, $S_{*}$ and Roux, A., Interaction of particles with ion-cyclotron waves and magnetosonic waves. Observations from GEOS1 and GEOS2. Planet. Space Sci. 32, $1393,1984$.

Oraevski, V. N., Konikov, Yu. V. and Khazanov, G. V., Transport Processes in Anisotropic Near-Terrestrial Plasma. Nauka, Moscow, 1985.

Tsytovich, V. N., The Theory of Turblent Plasma. Atomizdat, Moscow, 1971

Young, D. T., Perraut, S., Roux, A., de Villedary, C., Gendrin, R., Korth, A., Kremser, G. and Jones, D., Wave-particle interaction near $\Omega_{\mathrm{Hc}}$ observed on GEOS1 and 2. Propa* gation of ion-cyclotron waves in $\mathrm{He}^{+}$-rich plasma. J. geophys. Res. 86, 6755, 1981

\section{Appendix}

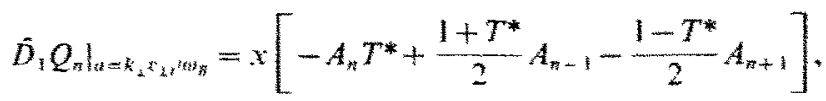

$$
\begin{aligned}
& \left.\hat{D}_{2} Q_{n}\right|_{w \rightarrow h_{1} n_{1}, n_{n}, n_{n}}=x\left[2(x-1) A_{n}-x\left(A_{n-1}+A_{n+1}\right)\right],
\end{aligned}
$$

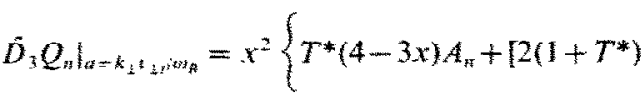

$$
\begin{aligned}
& \left.-\left(2 T^{*}+1\right) x\right] A_{n-1}+\left[2\left(1-T^{*}\right)-\left[2 T^{*}-1\right) x\right] A_{n+1} \\
& \left.-\frac{1+T^{*}}{2} \times A_{n-2}-\frac{1-T^{*}}{2} \times A_{n+2}\right\}
\end{aligned}
$$


K. V. Gamayunov et al.: Plasma hydrodynamics in view of quasilinear effects

33

$$
\begin{aligned}
& \left.\hat{D}_{1}\left(Q_{n} \frac{n}{x}+T R_{n}\right)\right|_{a=k_{1} E_{1 ;}\left(\omega_{3}\right.}=\frac{x}{2}\left\{\left(3|T|^{2}-1\right) A_{n}\right. \\
& +\frac{1+T^{*}}{2}(1+T-2 x T) A_{n-1}-\frac{1-T^{*}}{2}(1-T+2 x T) A_{n+1} \\
& \left.+\frac{|1+T|^{2}}{4} x A_{n-2}+\frac{|1-T|^{2}}{4} x A_{n+2}\right\} \text {, } \\
& \left.\hat{D}_{2}\left(Q_{n} \frac{n}{x}+T R_{n}\right)\right|_{a=k_{L^{2}+t} ; \omega_{B}}=x\left\{T(4-3 x) A_{n}\right. \\
& +[2 T(x-1)+x-2] A_{n-1}+[2 T(x-1)-x+2] A_{n+1} \\
& \left.-\frac{1+T}{2} x A_{n-2}+\frac{1-T}{2} x A_{n+2}\right\} \\
& \left.\hat{D}_{3}\left(Q_{n} \frac{n}{x}+T R_{n}\right)\right|_{n=k_{1} n_{1}, \omega_{\theta}}=x\left\{(2-x)\left(1-5|T|^{2}\right) x A_{n}\right. \\
& +\left[-2|1+T|^{2}+x\left(1+4 T^{*}+4 T+7|T|^{2}\right)\right. \\
& \left.+\frac{x^{2}}{4}\left(1-5 T^{*}-5 T-15|T|^{2}\right)\right] A_{n-1}
\end{aligned}
$$

(A4)

$$
\begin{aligned}
& +\left[-2|1-T|^{2}+x\left(1-4 T^{*}-4 T+7|T|^{2}\right)\right. \\
& \left.+\frac{x^{2}}{4}\left(1+5 T^{*}+5 T-15|T|^{2}\right)\right] A_{n+1} \\
& +x\left[-2|1+T|^{2}+\frac{x^{2}}{2}\left(1+2 T^{*}+2 T+3|T|^{2}\right)\right] A_{n-2} \\
& +x\left[-2|1-T|^{2}+\frac{x}{2}\left(1-2 T^{*}-2 T+3|T|^{2}\right)\right] A_{n+2} \\
& \left.-\frac{x^{2}}{4}|1+T|^{2} A_{n \cdot 3}-\frac{x^{2}}{4}|1-T|^{2} A_{n+3}\right\},
\end{aligned}
$$

(Ab)

where:

$$
\hat{D}_{1}=T^{*} \dot{\lambda} \frac{\partial}{\partial a}+n ; \quad \hat{D}_{2}=\lambda^{2} \frac{\partial^{2}}{\partial a^{2}}+\lambda \frac{\partial}{\partial a}-n^{2}
$$

and :

$$
\hat{D}_{3}=T^{*}\left(\lambda^{3} \frac{\partial^{3}}{\partial a^{3}}+\lambda^{2} \frac{\partial^{2}}{\partial a^{2}}-\left(1+n^{2}\right) \lambda \frac{\partial}{\partial a}+2 n^{2}\right)+n \hat{D}_{2}
$$

